\title{
Influence of Accelerated Temperature on Thermal Stability of Inactivated Oil Based Vaccines
}

\author{
Muhammad Sarmad ${ }^{1}$, Muhammad Danish Mehmood², Huma Anwar², Muhammad Usman Ghani², \\ Khusbu Farwa ${ }^{1} \&$ Saira Munir ${ }^{3}$ \\ ${ }^{1}$ Institute of Molecular Biology and Biotechnology, University of Lahore, Pakistan \\ ${ }^{2}$ Ottoman Pharma (Immuno Division), Raiwind Road Lahore, Pakistan \\ ${ }^{3}$ Institute of Microbiology, University of Agriculture, Faisalabad \\ Correspondence: Muhammad Danish Mehmood, Director Technical's at Ottoman Pharma Immuno Division, \\ Lahore, Pakistan. E-mail: drdanishmehmood@gmail.com
}

Received: August 30, 2019

Accepted: September 12, 2019

Online Published: September 30, 2019

doi:10.5539/ijb.v11n4p101

URL: https://doi.org/10.5539/ijb.v11n4p101

\begin{abstract}
Accelerated thermal stability of vaccines during shelf life has vital impact on its potency. Temperature directly affects the stability of the vaccines and degradation of immunogen depends upon the storage and environmental conditions. The current study was undertaken to evaluate the potency of three different inactivated oil based commercial vaccines under artificially induced thermal conditions such as $4{ }^{\circ} \mathrm{C}, 24^{\circ} \mathrm{C}, 36^{\circ} \mathrm{C}$ and $45^{\circ} \mathrm{C}$. The vaccines were analyzed for their viscosity, density, $\mathrm{pH}$, stability, particle distribution and serological potency after 10,20 and 30 days. It was revealed that vaccines stored for 30 days at $4^{\circ} \mathrm{C}$ upto $24^{\circ} \mathrm{C}$ showed significantly better physiochemical response and in vivo serological potency ( $p>0.05$ ) as compared to vaccines stored for 30 days at $36^{\circ} \mathrm{C}$ and $45^{\circ} \mathrm{C}(\mathrm{p}<0.05)$. Otto ND showed significantly better emulsion stability even under accelerated temperature at $45^{\circ} \mathrm{C}$ for 30 days $(\mathrm{p}>0.05)$ as compared to Gallimune and Medivac $(\mathrm{p}<0.05)$. The density of Medivac was stable under thermal stress conditions even at $36^{\circ} \mathrm{C}$ for 30 days $(\mathrm{p}>0.05)$ as compare to Otto ND and Gallimune vaccine $(\mathrm{p}<0.05)$.

It is concluded that inactivated oil based ND vaccines can resist elevated thermal stress up to $36^{\circ} \mathrm{C}$ for 30 days without any ill effect on physiochemical properties of emulsion and decline in serological potency. The vaccines showed significant deterioration physically at temperature $>30^{\circ} \mathrm{C}$ when stored for 30 days whereas, immunogenic response was protective with minimal uniformity. However, above vaccine could be used for effective immuneprophylaxis in poultry.
\end{abstract}

Keywords: Emulsion Accelerated Stability, Inactivated Oil Based Vaccines, Heamagglutination Inhibition Test, Cold Chain

\section{Introduction}

Vaccines are complex heterogeneous suspension of chemicals and microbiological agents that has been used for the immunization against a specific disease. Generally, a vaccine consists of proteins, carbohydrates, lipids and desired microorganisms. Live vaccines consist of attenuated or modified organisms, stabilizer and a preservative. Conversely, inactivated vaccines are composed of killed organism along with adjuvants and preservatives. These all components play a very important role in efficacy, potency and stability of a vaccine but they are highly sensitive to the environmental factors especially to the temperature (WHO, 2006).

In the case of liquid formulation of a vaccine, humidity is not a major issue but other environmental factor such as light (photo stability) is resolved by using the amber glass for the packing of light sensitive components (WHO, 2006). The ability of a vaccine to possess its physical, chemical, microbiological and biological properties in the specific limits during shelf life is referred as the stability of a vaccine. Shelf life is the time period in which a vaccine remains stable at recommended storage conditions (Garba et al., 1986).

There are two main categories of stability testing to determine the shelf life, real time stability and accelerated stability. Storage of vaccines at recommended storage condition is referred as the real time or real condition stability, while in the accelerated stability the product is stored at the elevated stress conditions (Fox, 2013). Accelerated and real time stability is used to define the degradation pattern which occurs when a vaccine fails to 
achieve the potential results. In 1980, smallpox was eradicated due to the availability of heat stable vaccine at that time (Evans et al., 2004). A major problem behind the slow eradication of polio infection is inadequate availability of cold chain storage for oral polio vaccines (Minor, 2012). A liquid formulation should be stable for 18-36 months when stored at $2-8^{\circ} \mathrm{C}$ and should be heat stable and can resist ambient temperature to allow short term storage and handling in the field. Vaccines should be stable be stable through multiple freeze thaw cycles and compatible with paternal injections in terms of tonicity (Evans et al., 2004). Adjuvants are used to enhance the stability of subunits vaccines because they are not immunogenic themselves and cannot evoke immune system for a long time interval. Currently, Oil based emulsions are alternate of aluminum salts adjuvants and show better immune response as compare to aluminum salts adjuvants (Roles, 2010).

In developing countries thermostability is major issue, the quality of the vaccine at risk due to the improper storage facilities, poor transportation and untrained manpower (Sarkar et al., 2003). Therefore, the purpose of this study is to develop various rapid and reliable tests to investigate the stability of inactivated oil based vaccine with reference to the international standards such as WHO, USP and EUP. For the purpose samples of different commercial vaccines from the local market were evaluated for their in house stability of emulsion and in vivo efficacy in broiler chicks through haemagglutination inhibition test.

\section{Materials and Methods}

The study was conducted in coordination with Ottoman Pharma, a licensed manufacturer of poultry vaccines located at Raiwind road, Lahore and University of Lahore. All the experiments were done in Research \& Development (R\&D) department of Ottoman Pharma, Lahore. The aim of the current study is to develop authentic method for the evaluation of oil based vaccines at different elevated temperatures.

\subsection{Source of Vaccines}

Three different brands of oil based inactivated vaccines such as Otto ND Flu Vac Ottoman Pharma Pakistan, Gallimune-Merial- France and Medivac-Hilton- Indonesia were purchased from their respective distributor and kept in cold chain till further use.

\subsection{Source of Birds for Efficacy Testing}

Total of 50 day old broilers were purchased from the well reputed hatchery of big bird poultry breeders located at Raiwind road and shifted immediately to the clean and fumigated experimental animal house of Ottoman Pharma. All the birds were reared under controlled conditions and offered with feed and water ad libitum.

\subsection{Experimental Design}

\subsubsection{Thermal Stability Testing}

5 vials of Otto ND Flu Vac, 5 vials of Gallimune and 3 vials of Medivac was transferred into 36 sub vials in such a way that each vial has $60 \mathrm{ml}$ of each vaccine as shown Figure 1A. Master vial of each vaccine was de-sealed in safety cabinet by wearing the sterile gloves as shown Figure 1B. Sub vials were properly labeled and transferred to their respective incubators with elevated temperature as $4{ }^{\circ} \mathrm{C}, 24^{\circ} \mathrm{C}, 36^{\circ} \mathrm{C}$ and $45^{\circ} \mathrm{C}$ for 30 days as shown Figure, 2. Samples were taken after 10, 20 and 30 days respectively and physiochemical properties of the vaccine were analyzed further details are shown in Table 1.

\subsubsection{Efficacy Test}

Total of 20 birds were divided into four groups each containing 4 birds. The birds were marked with specific color and immunized with respective vaccine at different time interval as shown in table. The blood of each bird of every group was collected from wing vein at 28 day post vaccination. The serum was extracted and subjected for haemagglutination inhibition test.

\subsubsection{Haemagglutination Inhibition Test}

96 well microtiter plate was filled with $50 \mu 1$ normal saline from 1-12 well with the help of micro pipette. $50 \mu 1$ of serum was dispensed in $1^{\text {st }}$ well and made 2 fold serial dilutions up to $10^{\text {th }}$ well of $1^{\text {st }}$ row. $50 \mu 1$ of $4 \mathrm{HA}$ antigen was added from 1 to $11^{\text {th }}$ well of $1^{\text {st }}$ row and the microtiter plate was incubated at $37^{\circ} \mathrm{C}$ for 20 minutes. After the incubation $50 \mu 1$ of $1 \%$ washes chicken RBCs was added from $1^{\text {st }}$ well to $12^{\text {th }}$ well of $1^{\text {st }}$ row. Results were recorded after 30 minutes.

\subsection{Statistical Analysis}

The data obtained in the study was analyzed by mean standard deviation and subsequently through repeated measure analysis of variables (ANOVA) using SPSS version 21. 
Table 1. Master Data Sheet of Vaccine's Label

\begin{tabular}{|c|c|c|c|}
\hline Sample & Label & Temperature & Days \\
\hline Otto ND Flu Vac & Otto ND Flu Vac/V1 & $4 \mathrm{C}$ & 10 \\
\hline Otto ND Flu Vac & Otto ND Flu Vac/V2 & $4 \mathrm{C}$ & 20 \\
\hline Otto ND Flu Vac & Otto ND Flu Vac/V3 & $4 \mathrm{C}$ & 30 \\
\hline Medivac & Medivac/V1 & $4 \mathrm{C}$ & 10 \\
\hline Medivac & Medivac/V2 & $4 \mathrm{C}$ & 20 \\
\hline Medivac & Medivac/V3 & $4 \mathrm{C}$ & 30 \\
\hline Gallimune & Gallimune/V1 & $4 \mathrm{C}$ & 10 \\
\hline Gallimune & Gallimune/V2 & $4 \mathrm{C}$ & 20 \\
\hline Gallimune & Gallimune/V3 & $4 \mathrm{C}$ & 30 \\
\hline Otto ND Flu Vac & Otto ND Flu Vac/V1 & $24 \mathrm{C}$ & 10 \\
\hline Otto ND Flu Vac & Otto ND Flu Vac/V2 & $24 \mathrm{C}$ & 20 \\
\hline Otto ND Flu Vac & Otto ND Flu Vac/V3 & $24 \mathrm{C}$ & 30 \\
\hline Medivac & Medivac/V1 & $24 \mathrm{C}$ & 10 \\
\hline Medivac & Medivac/V2 & $24 \mathrm{C}$ & 20 \\
\hline Medivac & Medivac/V3 & $24 \mathrm{C}$ & 30 \\
\hline Gallimune & Gallimune/V1 & $24 \mathrm{C}$ & 10 \\
\hline Gallimune & Gallimune/V2 & $24 \mathrm{C}$ & 20 \\
\hline Gallimune & Gallimune/V3 & $24 \mathrm{C}$ & 30 \\
\hline Otto ND Flu Vac & Otto ND Flu Vac/V1 & $36 \mathrm{C}$ & 10 \\
\hline Otto ND Flu Vac & Otto ND Flu Vac/V2 & $36 \mathrm{C}$ & 20 \\
\hline Otto ND Flu Vac & Otto ND Flu Vac/V3 & $36 \mathrm{C}$ & 30 \\
\hline Medivac & Medivac/V1 & $36 \mathrm{C}$ & 10 \\
\hline Medivac & Medivac/V2 & $36 \mathrm{C}$ & 20 \\
\hline Medivac & Medivac/V3 & $36 \mathrm{C}$ & 30 \\
\hline Gallimune & Gallimune/V1 & $36 \mathrm{C}$ & 10 \\
\hline Gallimune & Gallimune/V2 & $36 \mathrm{C}$ & 20 \\
\hline Gallimune & Gallimune/V3 & $36 \mathrm{C}$ & 30 \\
\hline Otto ND Flu Vac & Otto ND Flu Vac/V1 & $45 \mathrm{C}$ & 10 \\
\hline Otto ND Flu Vac & Otto ND Flu Vac/V2 & $45 \mathrm{C}$ & 20 \\
\hline Otto ND Flu Vac & Otto ND Flu Vac/V3 & $45 \mathrm{C}$ & 30 \\
\hline Medivac & Medivac/V1 & $45 \mathrm{C}$ & 10 \\
\hline Medivac & Medivac/V2 & $45 \mathrm{C}$ & 20 \\
\hline Medivac & Medivac/V3 & $45 \mathrm{C}$ & 30 \\
\hline Gallimune & Gallimune/V1 & $45 \mathrm{C}$ & 10 \\
\hline Gallimune & Gallimune/V2 & $45 \mathrm{C}$ & 20 \\
\hline Gallimune & Gallimune/V3 & $45 \mathrm{C}$ & 30 \\
\hline
\end{tabular}
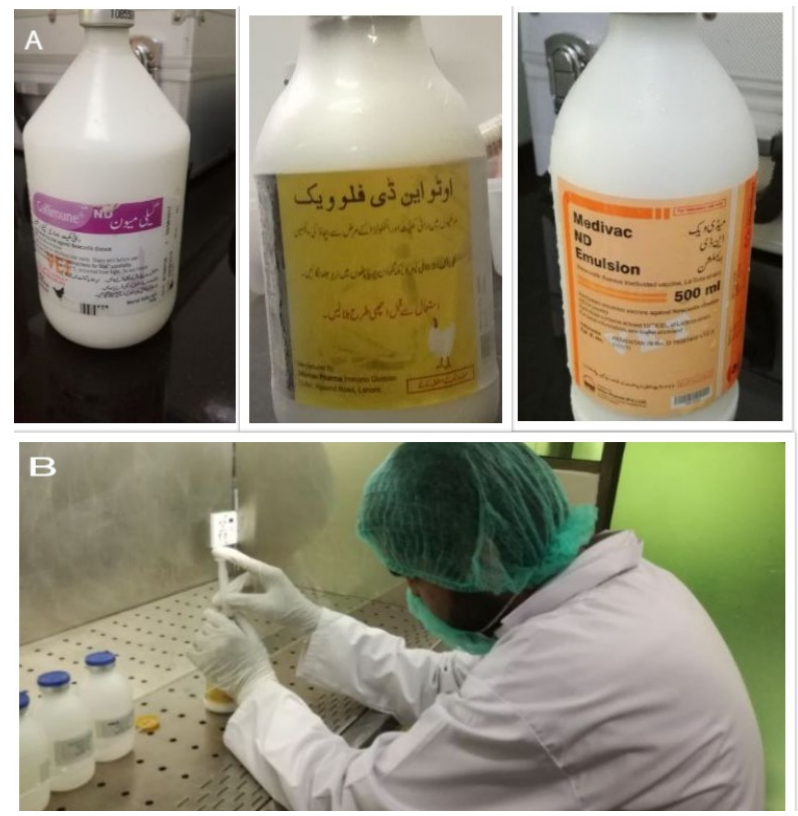

Figure 1. (A) ND Inactivated oil based vaccines (B) Vaccines are distributed in sub-vials 


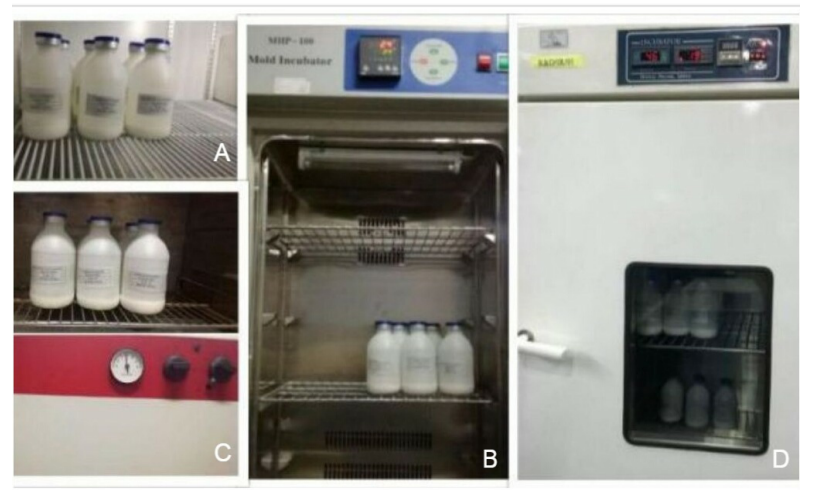

Figure 2. (A) vaccines stored at $4^{\circ} \mathrm{C}$ (B) vaccines stored at $24^{\circ} \mathrm{C}(\mathrm{C})$ vaccines stored at $36^{\circ} \mathrm{C}(\mathrm{D})$ vaccines stored at $45^{\circ} \mathrm{C}$

\section{Results}

Thermal stress on three different commercial vaccines such as Otto ND Flu Vac, Medivac and Galimune, were evaluated in the terms of change in their physiochemical properties.

Otto ND Flu vac documented detectable physiochemical changes when stored at $4{ }^{\circ} \mathrm{C}, 24^{\circ} \mathrm{C}, 36^{\circ} \mathrm{C}$ and $45^{\circ} \mathrm{C}$ for 30 days. At $4^{\circ} \mathrm{C}$ storage the $\mathrm{M} \pm \mathrm{SD}$ in viscosity, $\mathrm{pH}$, density, stability, particle distribution of emulsion and serological potency were $12.3 \pm .070,6.3 \pm .023,0.9 \pm .005,6.00 \pm .00,5.2 \pm 1.09$ and $25.6 \pm 8.76$ respectively. At $24^{\circ} \mathrm{C}$ storage the $\mathrm{M} \pm \mathrm{SD}$ in viscosity, density, $\mathrm{pH}$, stability, particle distribution of emulsion and serological potency were $9.6 \pm .005,0.88 \pm .011,6.4 \pm .007,6 \pm 0.00,4 \pm 0.00$ and $25.6 \pm 8.76$ respectively. At $36^{\circ} \mathrm{C}$ storage the $\mathrm{M} \pm \mathrm{SD}$ in viscosity, density, $\mathrm{pH}$, stability, particle distribution of emulsion and serological potency were $9.2 \pm .007,0.89 \pm .007$, $6.00 \pm .070,5.2 \pm 1.09,3.6 \pm 1.67$ and $19.20 \pm 12.13$ respectively. At $45^{\circ} \mathrm{C}$ storage the $\mathrm{M} \pm \mathrm{SD}$ in viscosity, density, $\mathrm{pH}$, stability, particle distribution of emulsion and serological potency were $7.6 \pm .020,0.854 \pm .005,5.94 \pm .038,4.8 \pm 1.09$, $3.2 \pm 1.09$ and $14.4 \pm 10.43$ respectively. Physiochemical and serological changes are shown in Figure 11, 15, 19, 23 and 27 respectively.

Medivac showed detectable physiochemical changes when stored at $4{ }^{\circ} \mathrm{C}, 24^{\circ} \mathrm{C}, 36^{\circ} \mathrm{C}$ and $45^{\circ} \mathrm{C}$ for 30 days. At $4^{\circ} \mathrm{C}$ storage the $\mathrm{M} \pm \mathrm{SD}$ in viscosity, $\mathrm{pH}$, density, stability, particle distribution of emulsion and serological potency were $13.69 \pm .07,6.04 \pm .089,0.88 \pm 0.013,6.00 \pm .00,4.4 \pm .89$ and $25.6 \pm 8.76$ respectively. At $24^{\circ} \mathrm{C}$ storage the $\mathrm{M} \pm \mathrm{SD}$ in viscosity, density, $\mathrm{pH}$, stability, particle distribution of emulsion and serological potency were $9.54 \pm .089$, $0.88 \pm .007,6.06 \pm .054,6 \pm 0.00,4 \pm 0.00$ and $22.4 \pm 8.76$ respectively. At $36^{\circ} \mathrm{C}$ storage the $\mathrm{M} \pm \mathrm{SD}$ in viscosity, density,

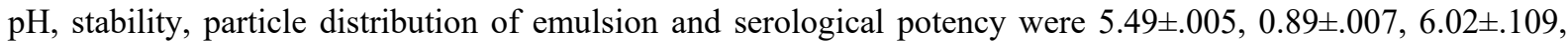
$4.4 \pm .894,3.2 \pm 1.09$ and $12.8 \pm 4.38$ respectively. At $45^{\circ} \mathrm{C}$ storage the $\mathrm{M} \pm \mathrm{SD}$ in viscosity, density, $\mathrm{pH}$, stability, particle distribution of emulsion and serological potency were $4.93 \pm .010,0.866 \pm .005,5.69 \pm .004,3.6 \pm .894$, $2.8 \pm 1.09$ and $11.2 \pm 4.38$ respectively. Physiochemical and serological changes are shown in Figure 11, 15, 19, 23 and 27 respectively.

Gallimune displayed detectable physiochemical changes when stored at $4{ }^{\circ} \mathrm{C}, 24^{\circ} \mathrm{C}, 36^{\circ} \mathrm{C}$ and $45^{\circ} \mathrm{C}$ for 30 days. The set parameters such as viscosity, $\mathrm{pH}$, density, stability, particle distribution and serological potency were evaluated at different time and recorded as shown in table 4.1 . At $4^{\circ} \mathrm{C}$ storage the Mean standard deviation $(\mathrm{M} \pm \mathrm{SD})$ in viscosity, $\mathrm{pH}$, density, stability, particle distribution of emulsion and serological potency were $13.59 \pm .014$, $6.24 \pm .005, .88 \pm .004,6.00 \pm .00,4.4 \pm .89$ and $25.6 \pm 8.76$ respectively. At $24^{\circ} \mathrm{C}$ storage the Mean standard deviation $(\mathrm{M} \pm \mathrm{SD})$ in viscosity, density, $\mathrm{pH}$, stability, particle distribution of emulsion and serological potency were $8.04 \pm .054,0.88 \pm .007,5.96 \pm .054,6 \pm 0.00,4 \pm 0.00$ and $22.4 \pm 8.76$ respectively. At $36^{\circ} \mathrm{C}$ storage the $\mathrm{M} \pm \mathrm{SD}$ in viscosity, density, $\mathrm{pH}$, stability, particle distribution of emulsion and serological potency were $5.9 \pm .007,0.87 \pm .007$, $6 \pm .070,4.8 \pm 1.09,3.2 \pm 1.09$ and $16.00 \pm 9.79$ respectively. At $45^{\circ} \mathrm{C}$ storage, the $\mathrm{M} \pm \mathrm{SD}$ in viscosity, density, $\mathrm{pH}$, stability, particle distribution of emulsion and serological potency were $5.17 \pm .405,0.864 \pm, 5.33 \pm .040,4.4 \pm .894$, $2.8 \pm 1.09$, and 12.8 \pm 4.38 respectively. Physiochemical and serological changes are shown in Figure 11, 15, 19, 23 and 27 respectively. 
Table 2. Effect of accelerated temperature at different time period on the stability of Otto ND Flu Vac

\begin{tabular}{|c|c|c|c|c|c|c|c|}
\hline \multirow{2}{*}{ Temperature } & \multirow{2}{*}{ Shelf Life } & \multicolumn{6}{|c|}{$\begin{array}{l}\text { Physiochemical Characteristics } \\
(\mathrm{M} \pm \mathrm{SD})=5\end{array}$} \\
\hline & & Viscosity & $\mathrm{pH}$ & Density & Stability & $\begin{array}{l}\text { Particle } \\
\text { Distribution }\end{array}$ & $\begin{array}{l}\text { Seroconversion } 28 \text { Day Post } \\
\text { Vaccination }\end{array}$ \\
\hline \multirow{3}{*}{$4^{\circ} \mathrm{C}$} & 10 & $18.06 \pm .054$ & $6.7 \pm .070$ & $0.91 \pm .007$ & $6 \pm 0.00$ & $6 \pm 0.00$ & $44.8 \pm 17.5$ \\
\hline & 20 & $13.89 \pm .01$ & $6.3 \pm .070$ & $0.9 \pm .007$ & $6 \pm 0.00$ & $6 \pm 0.00$ & $38.4 \pm 14.31$ \\
\hline & 30 & $12.3 \pm .070$ & $6.3 \pm .023$ & $0.9 \pm .005$ & $6 \pm 0.00$ & $5.2 \pm 1.09$ & $25.6 \pm 8.76$ \\
\hline \multirow{3}{*}{$24^{\circ} \mathrm{C}$} & 10 & $13.3 \pm .070$ & $6.5 \pm .070$ & $0.896 \pm .011$ & $6 \pm 0.00$ & $6 \pm 0.00$ & $38.4 \pm 14.31$ \\
\hline & 20 & $10.90 \pm .008$ & $6.45 \pm .070$ & $0.89 \pm .007$ & $6 \pm 0.00$ & $6 \pm 0.00$ & $32.0 \pm 0.00$ \\
\hline & 30 & $9.6 \pm .005$ & $6.4 \pm .007$ & $0.88 \pm .011$ & $6 \pm 0.00$ & $4 \pm 0.00$ & $25.6 \pm 8.76$ \\
\hline \multirow{3}{*}{$36^{\circ} \mathrm{C}$} & 10 & $10.9 \pm .070$ & $6.5 \pm .070$ & $0.9 \pm .007$ & $6 \pm 0.00$ & $6 \pm 0.00$ & $38.4 \pm 14.31$ \\
\hline & 20 & $10.4 \pm .007$ & $6.19 \pm .017$ & $0.90 \pm .004$ & $6 \pm 0.00$ & $6 \pm 0.00$ & $22.4 \pm 8.76$ \\
\hline & 30 & $9.2 \pm .007$ & $6 \pm .070$ & $0.89 \pm .007$ & $5.2 \pm 1.09$ & $3.6 \pm 1.67$ & $19.20 \pm 12.13$ \\
\hline \multirow{3}{*}{$45^{\circ} \mathrm{C}$} & 10 & $10.51 \pm .026$ & $6.4 \pm .018$ & $0.892 \pm .008$ & $6 \pm 0.00$ & $6 \pm 0.00$ & $28.8 \pm 7.15$ \\
\hline & 20 & $10.08 \pm .092$ & $6.06 \pm .046$ & $0.866 \pm .005$ & $6 \pm 0.00$ & $5.2 \pm 1.09$ & $17.6 \pm 8.76$ \\
\hline & 30 & $7.6 \pm .020$ & $5.94 \pm .038$ & $0.854 \pm .005$ & $4.8 \pm 1.09$ & $3.2 \pm 1.09$ & $14.4 \pm 10.43$ \\
\hline
\end{tabular}

Table 3. Effect of accelerated temperature at different time period on the stability of Medivac

\begin{tabular}{|c|c|c|c|c|c|c|c|c|c|c|}
\hline \multirow{2}{*}{ Temperature } & \multirow{2}{*}{ Shelf Life } & \multicolumn{9}{|c|}{$\begin{array}{l}\text { Physiochemical Characteristics } \\
(\mathrm{M} \pm \mathrm{SD})=5\end{array}$} \\
\hline & & Viscosity & $\mathrm{pH}$ & Density & Stability & $\begin{array}{l}\text { Particle } \\
\text { Distribution }\end{array}$ & $\begin{array}{l}\text { Seroconversion } \\
\text { Vaccination }\end{array}$ & 28 & Day & Post \\
\hline \multirow{3}{*}{$4^{\circ} \mathrm{C}$} & 10 & $13.88 \pm .044$ & $6.5 \pm .070$ & $.91 \pm .007$ & $6 \pm 0.00$ & $6 \pm 0.00$ & $32 \pm 0.00$ & & & \\
\hline & 20 & $11.5 \pm .008$ & $6 \pm .070$ & $0.88 \pm .008$ & $6 \pm 0.00$ & $6 \pm 0.00$ & $32 \pm 0.00$ & & & \\
\hline & 30 & $13.69 \pm .07$ & $6.04 \pm .089$ & $.88 \pm 0.013$ & $6 \pm 0.00$ & $5.2 \pm 1.09$ & $25.6 \pm 8.76$ & & & \\
\hline \multirow{3}{*}{$24^{\circ} \mathrm{C}$} & 10 & $10.2 \pm .070$ & $6 \pm .070$ & $0.9 \pm 0.005$ & $6 \pm 0.00$ & $6 \pm 0.00$ & $32.0 \pm 0.0$ & & & \\
\hline & 20 & $9.9 \pm .007$ & $5.9 \pm .007$ & $0.9 \pm .007$ & $6 \pm 0.00$ & $6 \pm 0.00$ & $32.0 \pm 0.0$ & & & \\
\hline & 30 & $9.54 \pm .089$ & $6.06 \pm .054$ & $0.88 \pm .007$ & $6 \pm 0.00$ & $4 \pm 0.00$ & $22.4 \pm 8.76$ & & & \\
\hline \multirow{3}{*}{$36^{\circ} \mathrm{C}$} & 10 & $9.7 \pm .070$ & $6.24 \pm .336$ & $0.9 \pm .007$ & $6 \pm 0.00$ & $6 \pm 0.00$ & $32.0 \pm 0.0$ & & & \\
\hline & 20 & $7.49 \pm .005$ & $6.3 \pm .005$ & $0.89 \pm .004$ & $6 \pm 0.00$ & $6 \pm 0.00$ & $22.4 \pm 8.76$ & & & \\
\hline & 30 & $5.49 \pm .005$ & $6.02 \pm .109$ & $0.89 \pm .007$ & $4.4 \pm .894$ & $3.2 \pm 1.09$ & $12.8 \pm 4.38$ & & & \\
\hline \multirow{3}{*}{$45^{\circ} \mathrm{C}$} & 10 & $8.95 \pm .054$ & $5.77 \pm .017$ & $0.888 \pm .004$ & $6 \pm 0.00$ & $6 \pm 0.00$ & $22.4 \pm 8.76$ & & & \\
\hline & 20 & $6.97 \pm .047$ & $5.74 \pm .007$ & $0.876 \pm .005$ & $5.2 \pm 1.09$ & $4.8 \pm 1.09$ & $19.2 \pm 7.15$ & & & \\
\hline & 30 & $4.93 \pm .010$ & $5.69 \pm .004$ & $0.866 \pm .005$ & $3.6 \pm .894$ & $2.8 \pm 1.09$ & $11.2 \pm 4.38$ & & & \\
\hline
\end{tabular}

Table 4. Effect of accelerated temperature at different time period on the stability of Gallimune

\begin{tabular}{|c|c|c|c|c|c|c|c|}
\hline \multirow{2}{*}{ Temperature } & \multirow{2}{*}{ Shelf Life } & \multicolumn{6}{|c|}{$\begin{array}{l}\text { Physiochemical Characteristics } \\
(\mathrm{M} \pm \mathrm{SD})=5\end{array}$} \\
\hline & & Viscosity & $\mathrm{pH}$ & Density & Stability & $\begin{array}{l}\text { Particle } \\
\text { Distribution }\end{array}$ & $\begin{array}{l}\text { Seroconversion } 28 \text { day post } \\
\text { vaccination }\end{array}$ \\
\hline \multirow{3}{*}{$4^{\circ} \mathrm{C}$} & 10 & $13.90 \pm .004$ & $6.3 \pm .070$ & $0.9 \pm .007$ & $6 \pm 0.00$ & $6 \pm 0.00$ & $44.8 \pm 17.5$ \\
\hline & 20 & $13.70 \pm 0.70$ & $6.00 \pm .070$ & $.88 \pm 0.007$ & $6.00 \pm 0.00$ & $6.00 \pm 0.00$ & $38.4 \pm 14.31$ \\
\hline & 30 & $13.59 \pm .014$ & $6.24 \pm .005$ & $.88 \pm .004$ & $6.00 \pm .00$ & $4.4 \pm .89$ & $25.6 \pm 8.76$ \\
\hline \multirow{3}{*}{$24^{\circ} \mathrm{C}$} & 10 & $12.3 \pm .070$ & $6.7 \pm .070$ & $0.88 \pm .013$ & $6 \pm 0.00$ & $6 \pm 0.00$ & $38.4 \pm 14.31$ \\
\hline & 20 & $8.7 \pm .070$ & $6 \pm .070$ & $0.88 \pm .007$ & $6 \pm 0.00$ & $6 \pm 0.00$ & $32 \pm 0.00$ \\
\hline & 30 & $8.04 \pm .054$ & $5.96 \pm .054$ & $0.88 \pm .005$ & $6 \pm 0.00$ & $4 \pm 0.00$ & $22.4 \pm 8.76$ \\
\hline \multirow{3}{*}{$36^{\circ} \mathrm{C}$} & 10 & $8 \pm .070$ & $6.5 \pm .070$ & $0.88 \pm .007$ & $6 \pm 0.00$ & $6 \pm 0.00$ & $38.4 \pm 14.31$ \\
\hline & 20 & $7 \pm .158$ & $6.23 \pm .021$ & $0.88 \pm .014$ & $6 \pm 0.00$ & $6 \pm 0.00$ & $25.6 \pm 8.76$ \\
\hline & 30 & $5.9 \pm .007$ & $6 \pm .070$ & $0.87 \pm .007$ & $4.8 \pm 1.09$ & $3.2 \pm 1.09$ & $16.00 \pm 9.79$ \\
\hline \multirow{3}{*}{$45^{\circ} \mathrm{C}$} & 10 & $7.032 \pm .049$ & $6.02 \pm .045$ & $0.878 \pm .008$ & $6 \pm 0.00$ & $6 \pm 0.00$ & $25.6 \pm 8.76$ \\
\hline & 20 & $6.46 \pm .017$ & $5.68 \pm .020$ & $0.872 \pm .008$ & $5.2 \pm 1.09$ & $4.8 \pm 1.09$ & $17.6 \pm 8.76$ \\
\hline & 30 & $5.17 \pm .405$ & $5.33 \pm .040$ & $0.864 \pm .005$ & $4.4 \pm .894$ & $2.8 \pm 1.09$ & $12.8 \pm 4.38$ \\
\hline
\end{tabular}




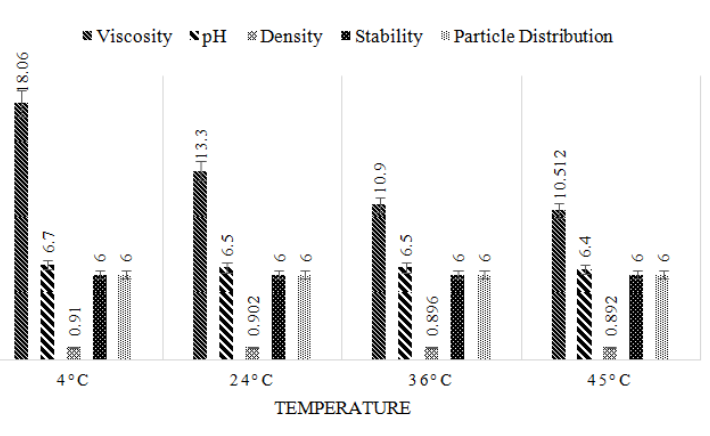

Figure 3. Accelerated thermal influence after 10 days at different temperatures on the physiochemical properties of Otto ND Flu Vac

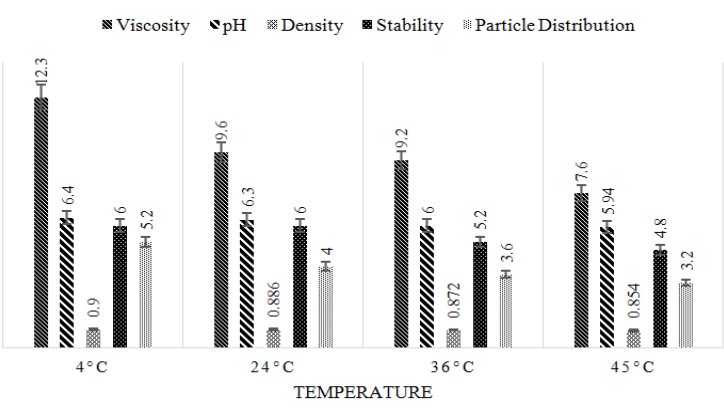

Figure 5. Accelerated thermal influence after 30 days at different temperatures on the physiochemical properties of Otto ND Flu Vac

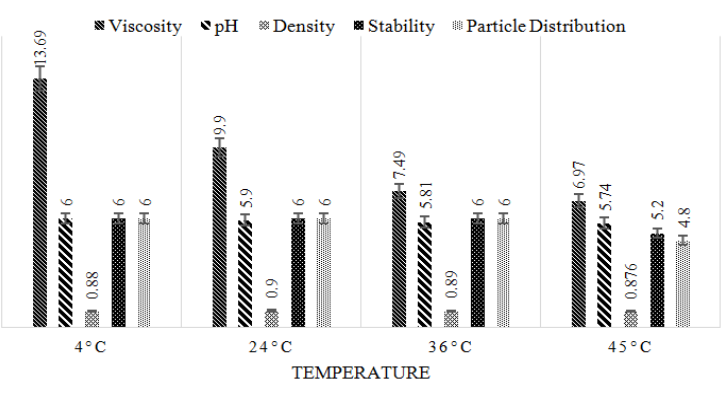

Figure 7. Accelerated thermal influence after 20 days at different temperatures on the Physiochemical properties of Medivac

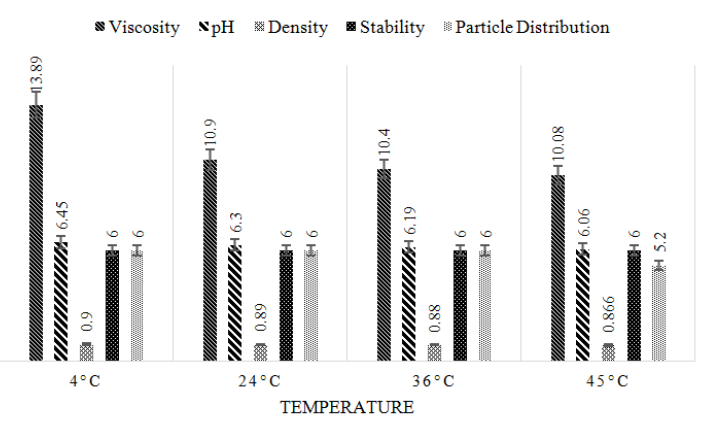

Figure 4. Accelerated thermal influence after 20 days at different temperatures on the physiochemical properties of Otto ND Flu Vac

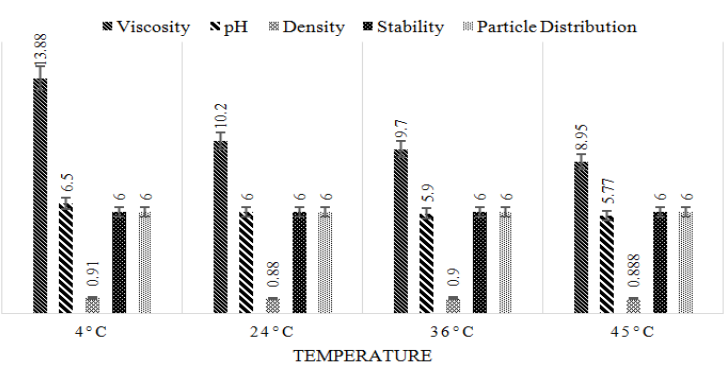

Figure 6. Accelerated thermal influence after 10 days at different temperatures on the physiochemical properties of Medivac

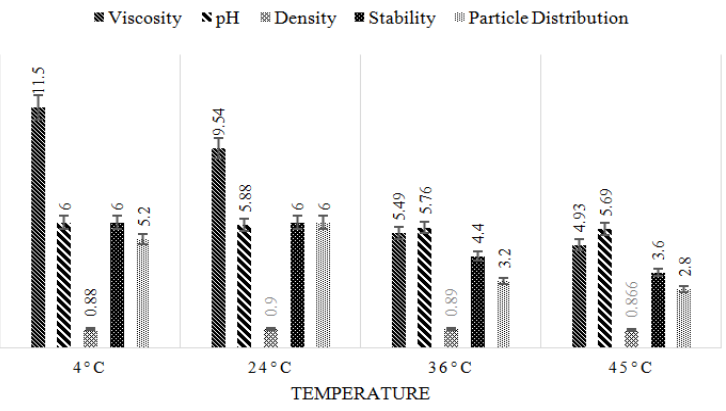

Figure 8. Accelerated thermal influence after 30 days at different temperatures on the physiochemical properties of Medivac 


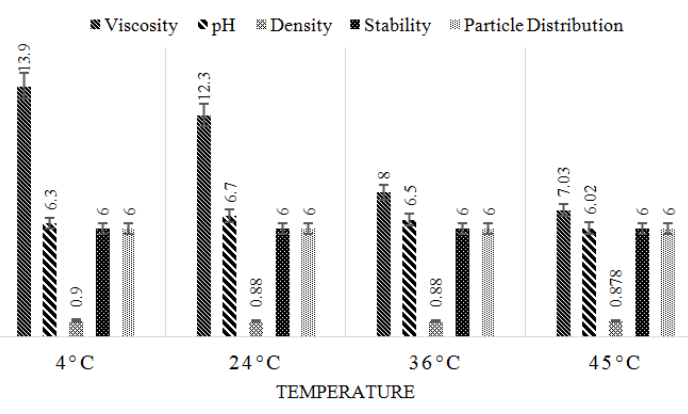

Figure 9. Accelerated thermal influence after 10 days on the physiochemical properties of Gallimune

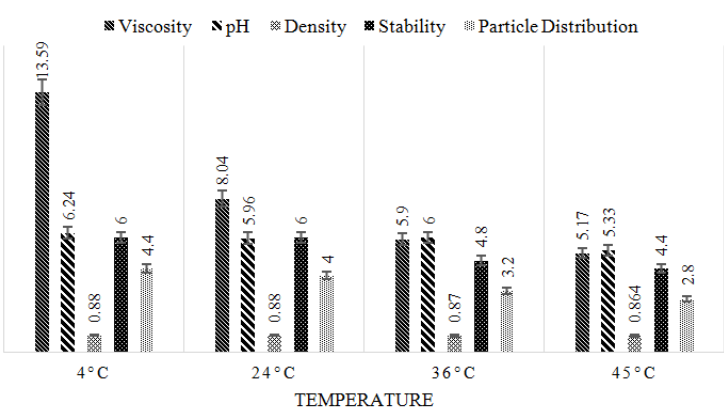

Figure 11. Accelerated thermal influence after 30 days at different temperatures on the physiochemical properties of Gallimune

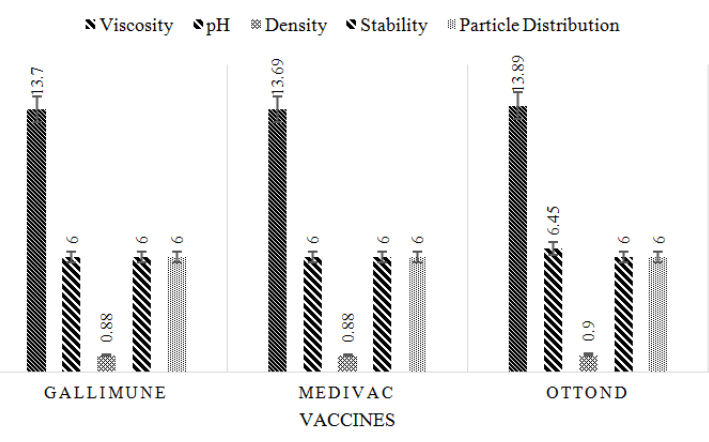

Figure 13. Effect of $4^{\circ} \mathrm{C}$ to 20D storage on physiochemical properties of killed vaccine

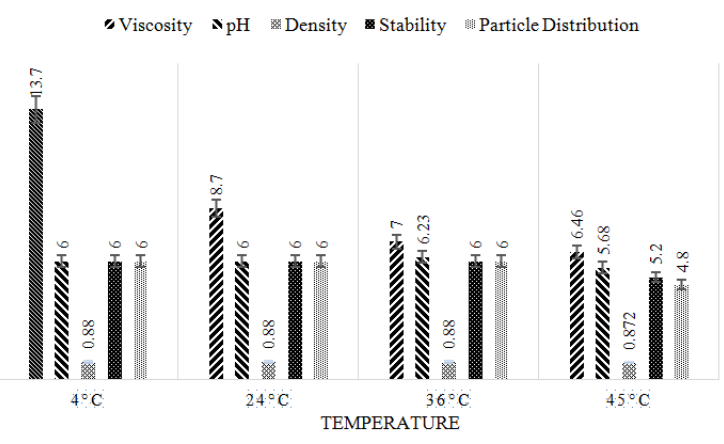

Figure 10. Accelerated thermal influence after 20 days on the physiochemical properties of Gallimune at different temperatures

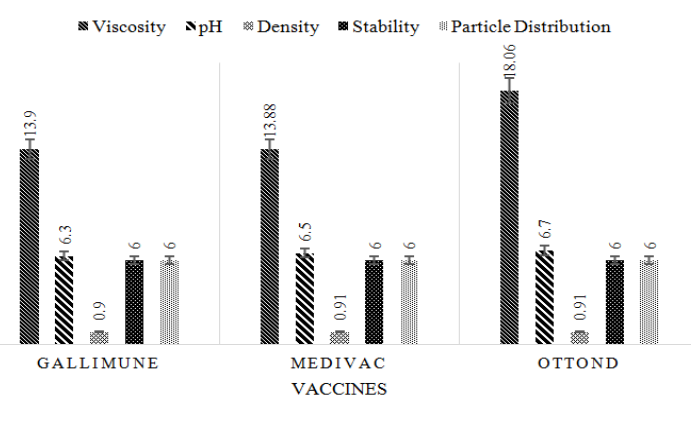

Figure 12. Effect of $4^{\circ} \mathrm{C}$ to $10 \mathrm{D}$ storage on physiochemical properties of killed vaccine

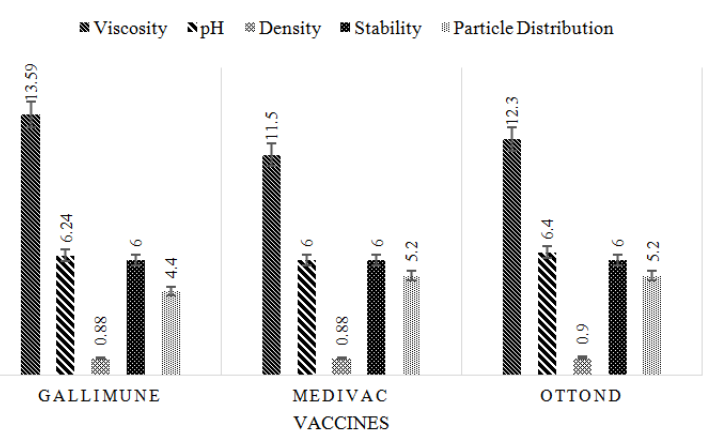

Figure 14. Effect of $4^{\circ} \mathrm{C}$ to $30 \mathrm{D}$ storage on physiochemical properties of killed vaccine 


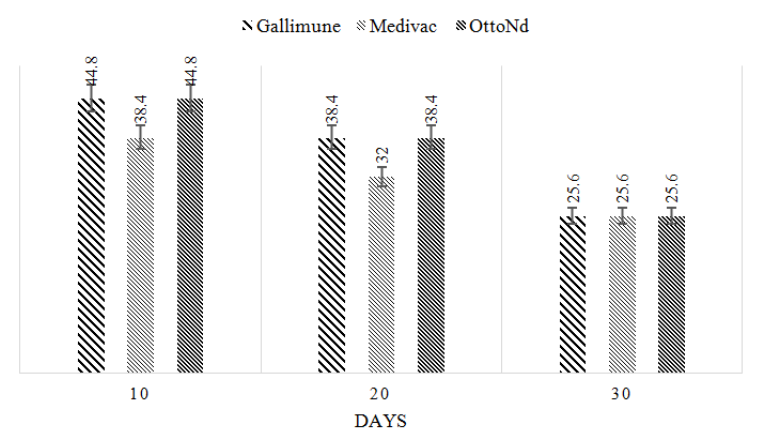

Figure 15. Accelerated thermal effect on serological potency of the vaccines at $4^{\circ} \mathrm{C}$

Viscosity $\mathbf{s}$ pH Density Stability MParticleDistribution

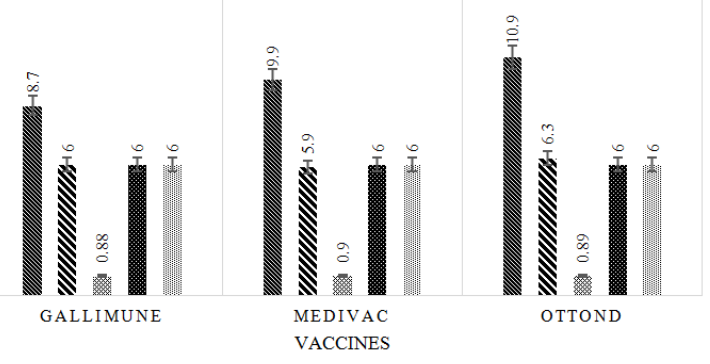

Figure 17. Effect of $24^{\circ} \mathrm{C}$ to $20 \mathrm{D}$ storage on physiochemical properties of killed vaccine

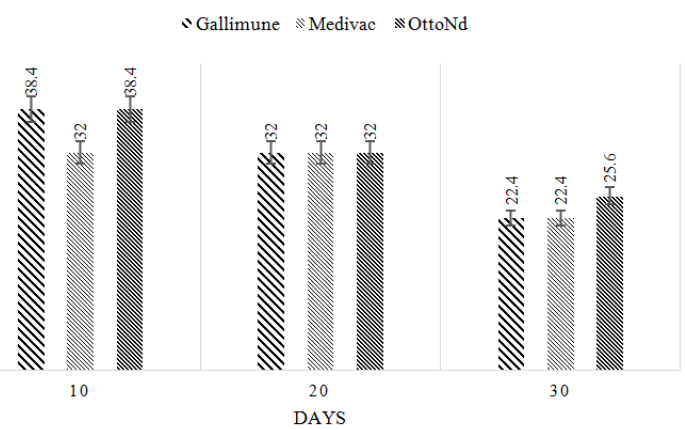

Figure 19. Accelerated thermal effect on serological potency of the vaccines at $24^{\circ} \mathrm{C}$

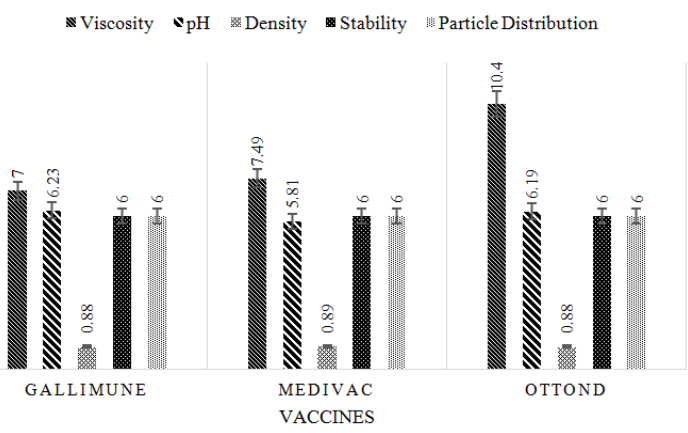

Figure 21. Effect of $36^{\circ} \mathrm{C}$ to $20 \mathrm{D}$ storage on physiochemical properties of killed vaccine

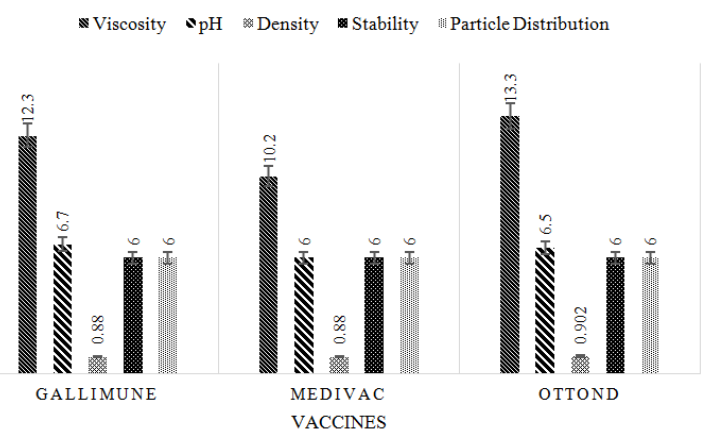

Figure 16. Effect of $24^{\circ} \mathrm{C}$ to $10 \mathrm{D}$ storage on physiochemical properties of killed vaccine

Niscosity $\mathbf{s}_{\mathrm{pH}}$ Density Stability il Particle Distribution

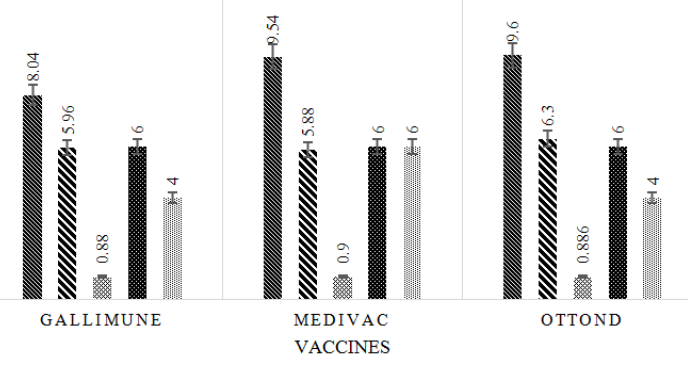

Figure 18. Effect of $24^{\circ} \mathrm{C}$ to 30D storage on physiochemical properties of killed vaccine

Niscosity $\mathbf{p H}$ Density Stability Particle Distribution

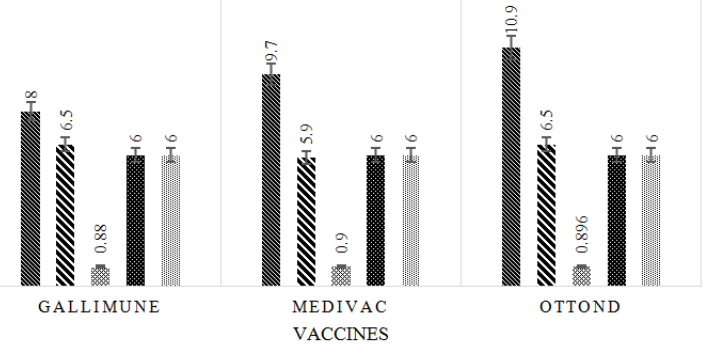

Figure 20. Effect of $36^{\circ} \mathrm{C}$ to $10 \mathrm{D}$ storage on physiochemical properties of killed vaccine

viscosity $\mathbf{s} \mathrm{pH}$ Density Stability Particle Distribution

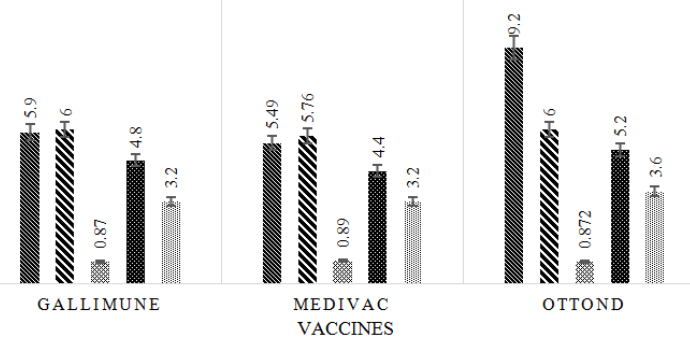

Figure 22. Effect of $36^{\circ} \mathrm{C}$ to $30 \mathrm{D}$ storage on physiochemical properties of killed vaccine 


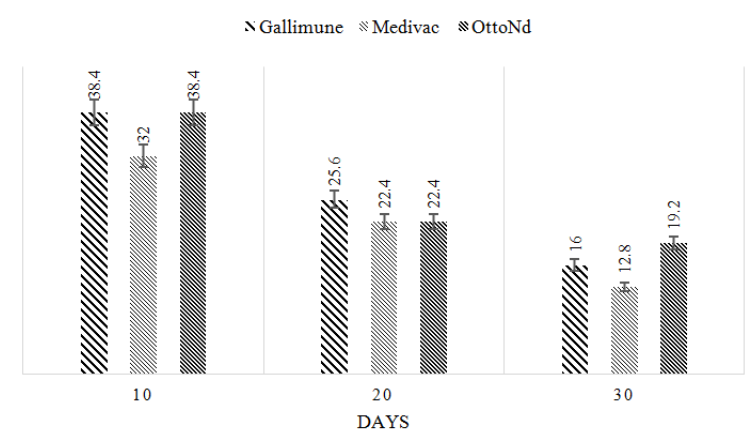

Figure 23. Accelerated thermal effect on serological potency of the vaccines at $36^{\circ} \mathrm{C}$

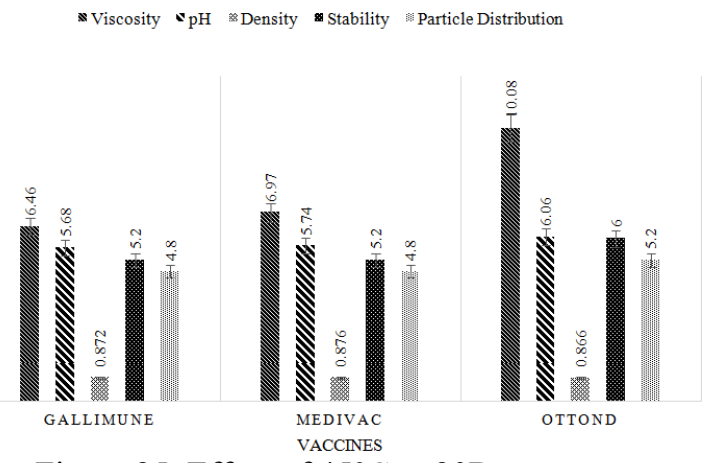

Figure 25. Effect of $45^{\circ} \mathrm{C}$ to 20D storage on physiochemical properties of killed vaccine

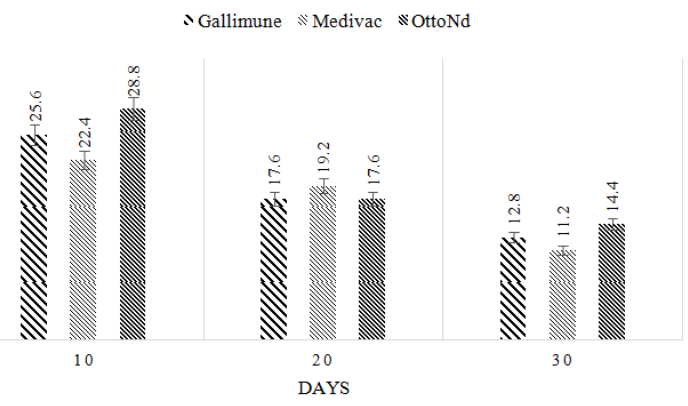

Figure 27. Accelerated thermal effect on serological potency of the vaccines at $45^{\circ} \mathrm{C}$

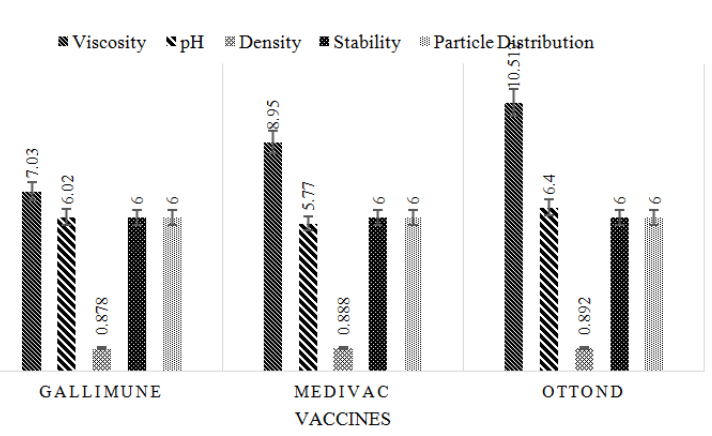

Figure 24. Effect of $45^{\circ} \mathrm{C}$ to $10 \mathrm{D}$ storage on physiochemical properties of killed vaccine

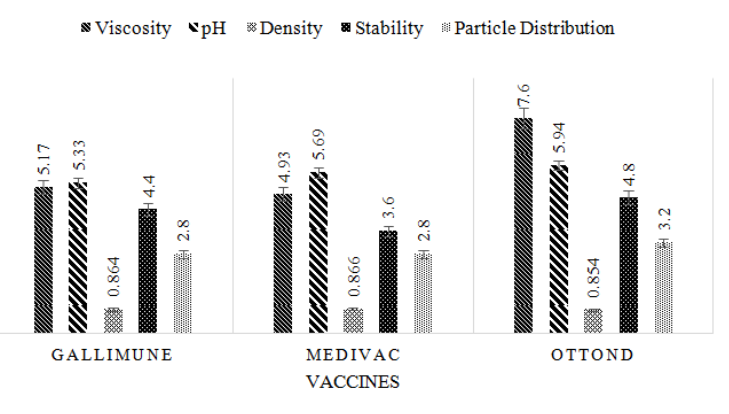

Figure 26. Effect of $45^{\circ} \mathrm{C}$ to 30D storage on physiochemical properties of killed vaccine

\section{Discussion}

Vaccines provoke the immune system by stimulating humoral and cell mediated responses against the infection. Mainly vaccines are consist of killed, attenuated or subunits of organism (Saroja et al., 2011). Killed and subunits vaccines are mainly associated with adjuvants and require boosters to enhance the immune response for long time interval (Bastola et al., 2017). Adjuvants are complex in nature and reduce the delivery of immunogen and also enhance the potency of vaccine by protecting the immunogen from the environmental factors. The contents of a vaccine are very sensitive to heat, light and radiations especially antigen so therefore to protect the antigen from environmental factors hence, most of the vaccines are kept in cold chain $(2-8)^{\circ} \mathrm{C}$. Potency of a vaccine is directly linked with maintenance of cold chain because fluctuations in temperature affect the physiochemical and biological properties of a vaccine (WHO, 2006). In current study the effect of temperature on stability of immunogen and physiochemical properties of killed oil based vaccines were documented. 
It was observed that viscosity, particle distribution, $\mathrm{pH}$ and density of Otto ND vaccine stored at $4^{\circ} \mathrm{C}$ for 30 days did not show any significant difference ( $p>0.05$ ) as compared to the vaccine stored at $24^{\circ} \mathrm{C}, 36^{\circ} \mathrm{C}$ and $45^{\circ} \mathrm{C}$ $(\mathrm{p}<0.05)$. It was experimentally ensured that particle distribution of Otto ND stored at $4^{\circ} \mathrm{C}, 24^{\circ} \mathrm{C}$ and $36^{\circ} \mathrm{C}$ for 30 days did not show any significant differences $(\mathrm{p}>0.05)$ as compared to Otto ND stored at $45^{\circ} \mathrm{C}$ for 30 days $(\mathrm{p}<0.05)$. In corresponding with Iyer et al., 2015, vaccine contained small particle size was more stable than the vaccine consist of large particle size and significant change $(p<0.05)$ in particle size was observed in vaccine stored at $40^{\circ} \mathrm{C}$ and $60^{\circ} \mathrm{C}$ for 30 days as compare to vaccine stored at $5^{\circ} \mathrm{C}$ and $25^{\circ} \mathrm{C}(\mathrm{p}>0.05)$. It was documented that stability of Otto ND vaccine stored at $4^{\circ} \mathrm{C}, 24^{\circ} \mathrm{C}, 36^{\circ} \mathrm{C}$ and $45^{\circ} \mathrm{C}$ for 30 days did not show any significant differences ( $>>0.05$ ). It was also observed that $\mathrm{HI}$ antibody titer of Otto ND vaccine stored at $4^{\circ} \mathrm{C}$ and $24^{\circ} \mathrm{C}$ for 30 days did not show any significant differences $(\mathrm{p}>0.05)$ as compared to Otto ND stored at $36^{\circ} \mathrm{C}$ and $45^{\circ} \mathrm{C}$ for 30 days $(\mathrm{p}<0.05)$.

It was observed that viscosity and $\mathrm{pH}$ of Medivac stored at $24^{\circ} \mathrm{C}, 36^{\circ} \mathrm{C}$ and $45^{\circ} \mathrm{C}$ for 30 days showed significant differences $(\mathrm{p}<0.05)$ as compared to the vaccine stored at $4^{\circ} \mathrm{C}$ for 30 days $(\mathrm{p}>0.05)$. It was documented that density, stability and particle distribution of Medivac stored at $45^{\circ} \mathrm{C}$ for 30 days showed significant difference $(\mathrm{p}<0.05)$ as compared to Medivac stored at $4^{\circ} \mathrm{C}, 24^{\circ} \mathrm{C}$ and $36^{\circ} \mathrm{C}$ for 30 days $(\mathrm{p}>0.05)$. The vaccines are stored at $23^{\circ} \mathrm{C}$ for 5 weeks showed minute decrease in the stability to that of vaccine stored at $55^{\circ} \mathrm{C}$ for 5 weeks showed conformational changes due to the elevated thermal stress Ho et al., 2000. It was experimentally confirmed that $\mathrm{HI}$ antibody titer of Medivac stored at $4^{\circ} \mathrm{C}$ and $24^{\circ} \mathrm{C}$ for 30 days did not show significant differences ( $p>0.05$ ) as compared to the vaccine stored at $36^{\circ} \mathrm{C}$ and $45^{\circ} \mathrm{C}$ for 30 days $(\mathrm{p}<0.05)$.

It was observed that viscosity of Gallimune when stored at $24^{\circ} \mathrm{C}, 36^{\circ} \mathrm{C}$ and $45^{\circ} \mathrm{C}$ for 30 days showed significant difference $(\mathrm{p}<0.05)$ as compared $4^{\circ} \mathrm{C}$ storage for 30 days $(\mathrm{p}>0.05)$. It was documented the density, $\mathrm{pH}$, viscosity and particle distribution of the vaccine stored at $4{ }^{\circ} \mathrm{C}$ and $24^{\circ} \mathrm{C}$ for 30 days did not show significant difference $(\mathrm{p}>0.05)$ when kept at $36^{\circ} \mathrm{C}$ and $45^{\circ} \mathrm{C}$ for 30 days $(\mathrm{p}<0.05)$. Whereas, $\mathrm{pH}$, stability and particle distribution of the vaccine stored at $4{ }^{\circ} \mathrm{C}, 24^{\circ} \mathrm{C}$, and $36^{\circ} \mathrm{C}$ for 30 days showed significant difference $(\mathrm{p}<0.05)$ to that of $45^{\circ} \mathrm{C}$ storage for 30 day ( $p>0.05$ ). It was confirmed that $\mathrm{HI}$ antibody titer of Gallimune vaccine stored at $45^{\circ} \mathrm{C}$ for 30 days showed significant difference $(\mathrm{p}<0.05)$ as compared to $4{ }^{\circ} \mathrm{C}, 24^{\circ} \mathrm{C}$ and $36^{\circ} \mathrm{C}$ storage for 30 days $(\mathrm{p}>0.05)$. Significant drop in humoral immune response of vaccine is Ho et al., 2000 observed when stored at $55^{\circ} \mathrm{C}$ for 5 weeks due to extreme thermal stress.

The results of the current study revealed that Gallimune, Medivac and Otto ND inactivated oil based vaccines found stable up to $24^{\circ} \mathrm{C}$ when stored for 30 days $(\mathrm{p}>0.05)$ whereas, Gallimune showed significantly better HI titer even at $36^{\circ} \mathrm{C}$ for 30 days $(p>0.05)$ as compare to Medivac and Otto ND vaccine $(p<0.05)$. Otto ND showed significantly better emulsion stability even under accelerated temperature at $45^{\circ} \mathrm{C}$ for 30 days $(\mathrm{p}>0.05)$ as compare to Gallimune and Medivac $(\mathrm{p}<0.05)$. The density of Medivac was stable under thermal stress conditions even at $36^{\circ} \mathrm{C}$ for 30 days ( $\left.p>0.05\right)$ as compare to Otto ND and Gallimune vaccine $(\mathrm{p}<0.05)$.

Thus it is concluded that all the vaccines used in the study showed optimum results at $2-10^{\circ} \mathrm{C}$ for 30 days. However, least physiochemical deterioration was recorded in each of the vaccine when stored between 10 to 30 days under thermal stress.

\section{Conflict of interests}

The authors declare that there is no conflict of interests regarding the publication of this paper.

\section{References}

Bastola, R., Noh, G., Keum, T., Bashyal, B., Seo, J., Choi, J., Oh, Y., Cho, Y., \& Lee, S. (2017). Vaccine adjuvants: smart components to boost the immune system. Arch. Pharma. Rese., 40, 1238-48.

Evans, R. K., Nawrocki, D. K., Isopi, L. A., Willimas, D. M., Casimiro, D. R., Chin, S., Chen, M., Zhu, D. M., Shiver, J. W., \& Volkin, D. B. (2004). Development of stable formulations for Adenovirus based vaccines. $J$. Pharm. Sci., 93, 2458-2475.

Fox, C. B., \& Haensler, J. (2013). An update on safety and immunogenicit of vaccines containing emulsion-based adjuvants. Expert Rev Vaccines, 12(7), 747-58.

Garba, S. A., \& Terry, R. J. (1986). Immunogenicity of oil based contagious bovine Pleuropneumonia vaccine in cattle. Vaccine, 4, 266-270.

Ho, M. M., Baligano, B., \& Corbel, M. J. (2000). Assessment of the stability and immunogenicity of meningococcal oligosaccharide C-CRM197 conjugate vaccine. Vaccine, 19, 716-725. 
Iyer, V., Cayatte, C., Guzman, B., Ohrum, S. K., Matuszak, R., Snell, A., Rajani, G. M., McCarthy, M. P., \& Muralidhara, B. (2015). Impact of formulatiuon and particle size on stability and immunogenicity of oil in water emulsion adjuvants. Human Vaccines \& Immunotherapeutics, 11(7), 1853-1864.

Minor, P. D. (2012). The polio-eradication programme and issues of the end games. J. Gen. Viro., 93, 457-474.

Roles, G. (2010). Unmet needs in modern Vaccinology: Adjuvants to improve the immune response. Vaccine, 28, 25-36.

Sarkar, J., Seernivas, B. P., Singh, R. P., Dhar, P., \& Bandyopadhyay, S. K. (2003). Comparative efficacy of various chemical stablier on the thermastability of live attenuated peste de petits ruminants (PPR) vaccine. Vaccine, 21, 4728-4735.

Saroja, Ch., Lakshmi, P., \& Bhaskaran, S. (2011). Recent trends in vaccine delivery systems. Int J. Pharma Investing, 1, 64-74.

World Health Organization. Guidelines on stability evaluation of vaccines. In. WHO Expert Committee on Biological Standardization. Fifty-second report. Geneva, World Health Organization, 2006.

\section{Copyrights}

Copyright for this article is retained by the author(s), with first publication rights granted to the journal.

This is an open-access article distributed under the terms and conditions of the Creative Commons Attribution license (http://creativecommons.org/licenses/by/4.0/). 